\title{
A $0.8-\mathrm{cm}$ clear cell neuroendocrine tumor G1 of the gallbladder with lymph node metastasis: a case report
}

\author{
Yuki Hirose ${ }^{1,2^{*}}$, Jun Sakata ${ }^{1}$, Kazuhiko Endo ${ }^{2}$, Masato Takahashi ${ }^{3}$, Reijiro Saito ${ }^{2}$, Hiroshi Imano ${ }^{2}$, Tomoki Kido², \\ Kei Yoshino ${ }^{2}$, Toshiki Sasaki ${ }^{3}$ and Toshifumi Wakai ${ }^{1}$
}

\begin{abstract}
Background: Neuroendocrine tumors (NETs) of the gallbladder are rare and generally considered low-grade malignancies. We herein describe a case of a patient with a 0.8-cm clear cell NET G1 of the gallbladder with nodal involvement.

Case presentation: A 65-year-old man with no medical history indicative of von Hippel-Lindau (VHL) disease underwent laparoscopic cholecystectomy for cholecystolithiasis. There was a 0.8-cm tumor in the neck of the gallbladder. Histologic examination revealed nests or trabecular growth of clear cells with small round-to-oval nuclei. Immunohistochemically, tumor cells showed positivity for chromogranin A and synaptophysin; Ki-67 index was $<1.0 \%$. Based on the World Health Organization 2010 classification, we made a diagnosis of clear cell variant of NET G1 without VHL disease. The tumor invaded the muscular layer and had no extension to the perimuscular connective tissue but had metastasized to a cystic duct node. A radical second resection with regional lymphadenectomy of the gallbladder was performed, and there was no metastasis on histology. After the definitive surgery, he was followed up for 10 months without adjuvant therapy and is alive and well with no evidence of recurrence.

Conclusions: Our experience suggests that, even when smaller than $1 \mathrm{~cm}, \mathrm{NET}$ G1 of the gallbladder can metastasize. When NET G1 is incidentally identified in the gallbladder of a surgical specimen, detailed pathologic examination of the cystic duct node, when found, should be performed to guide whether a radical second resection with regional lymphadenectomy is appropriate.
\end{abstract}

Keywords: Neuroendocrine tumor, Lymph node metastasis, Gallbladder, Surgical resection

\section{Background}

Neuroendocrine tumor (NET) is traditionally an umbrella term that encompasses a broad family of neoplasms originating from neural and endocrine structures located all over the body, especially in the gastrointestinal tract and lung $[1,2]$. Primary NETs of the gallbladder are rare and constitute $<1 \%$ of all NETs $[1,3]$. Clear cell NET of the gallbladder is even rarer; it has been described in association with von Hippel-Lindau (VHL) disease [4], which is a rare autosomal hereditary disease and generates

\footnotetext{
* Correspondence: y-hirose@med.niigata-u.ac.jp

${ }^{1}$ Division of Digestive and General Surgery, Niigata University Graduate

School of Medical and Dental Sciences, Niigata 951-8510, Japan

${ }^{2}$ Department of Digestive Surgery, Akita Kousei Medical Center, Akita, Japan

Full list of author information is available at the end of the article
}

tumors of the pancreas, kidneys, central nervous system, adrenal glands, and reproductive organs, but rarely of the biliary system [5].

The World Health Organization (WHO) 2010 classification divides these tumors into NET G1, G2, and G3 (or neuroendocrine carcinoma: NEC), based on the histologic evaluation of the proliferation rate and mitotic index of NET cells [6]. This classification enables prognostically relevant patient stratification, and NET G1 is thought to be a relatively "benign" category. Also, the risk of malignant behavior of NETs is widely accepted to increase in a size-dependent fashion [6-8]. Here, we describe a case of a patient who had a tiny clear cell NET G1 of the gallbladder with nodal involvement. 


\section{Case presentation}

A 64-year-old man presented to our hospital with epigastric pain. He exhibited no hormone-related symptoms, such as flushing, diarrhea, stomach aches, or hypoglycemia, and had no past or family history of VHL disease. On admission, the abdomen was nontender and laboratory data showed mild elevation of liver enzymes (AST, 474 IU/L; ALT, $231 \mathrm{IU} / \mathrm{L}$ ) and the white blood cell count $\left(11 \times 10^{3} / \mu \mathrm{L}\right)$. Abdominal ultrasonography showed the gallbladder and common bile duct stones and a low echoic nodule in the neck of the gallbladder preoperatively suspected as Rokitansky-Aschoff sinuses (Fig. 1). Contrast-enhanced abdominal computed tomography (CT) also revealed gallbladder and common bile duct stones but did not reveal a mass in the neck of the gallbladder. The patient underwent endoscopic sphincterotomy, and common bile duct stones were extracted successfully.

Almost 1 month after endoscopic treatment, laboratory data were within normal limits. We did not check the levels of tumor marker, urinary 5-hydroxyindoleacetic acid, or plasma serotonin because neither cancer nor NET was suspected at that time. He underwent laparoscopic cholecystectomy. Macroscopically, the specimen contained a yellowish submucosal nodule, located in the neck of the gallbladder, the size of which was $0.8 \times 0.8 \mathrm{~cm}$ (Fig. 2). Histologic examination revealed nests or trabecular growth of clear cells containing small round-to-oval nuclei with inconspicuous nucleoli (Fig. 3a) and showing no mitosis. The tumor surface was covered by intact epithelium. Immunohistochemically, tumor cells showed the expression of chromogranin A (Fig. 3b) and synaptophysin (Fig. 3c), and a Ki-67 index $<1.0 \%$ (Fig. 3d). Pathologic diagnosis of the

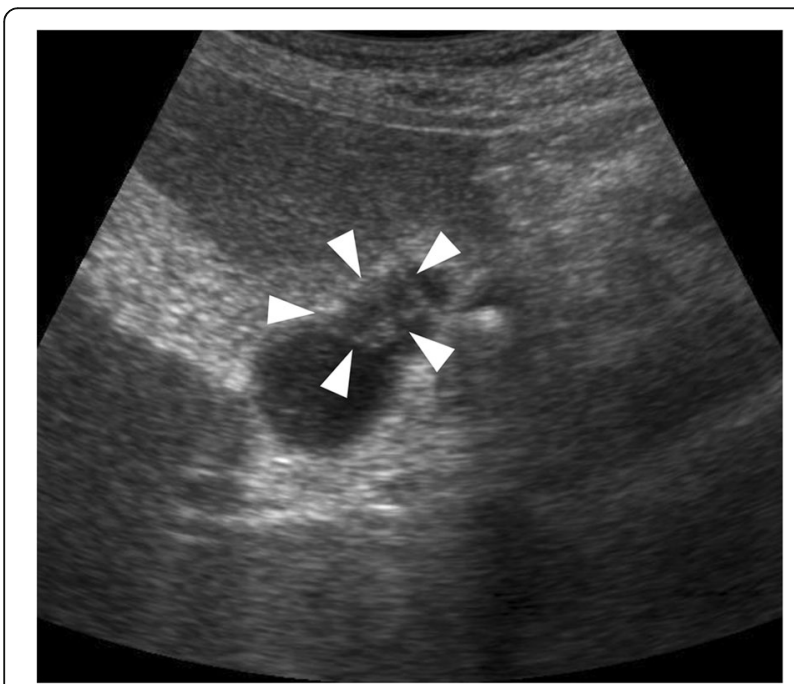

Fig. 1 Ultrasonography image shows a 0.8-cm low echoic nodule (arrowheads) in the neck of the gallbladder, which was preoperatively suspected to be a Rokitansky-Aschoff sinuses tumor was NET G1 according to the WHO 2010 classification and a clear cell variant without VHL disease. The tumor invaded the muscular layer and showed no extension to the perimuscular connective tissue, but had metastasized to a cystic duct node.

A radical second resection with regional lymphadenectomy of the gallbladder was performed, and histologically there was no metastasis. An R0 resection was confirmed, and he received no adjuvant therapy. After discharge, he was followed up every 1-3 months on an outpatient basis with physical examination, laboratory tests, and/or contrast-enhanced abdominal CT, which showed no evidence of recurrence. He remains alive and well 10 months after the definitive resection.

\section{Discussion}

Oberndorfer first described NETs and coined the term "carcinoid" (or "Karzinoide") in 1907 for these "presumptively" benign neoplasms [9]. Primary NETs of the gallbladder were first described by Joel in 1929. The majority of primary NETs occur in the gastrointestinal tract (66\%) [10], followed by the bronchopulmonary system (31\%), and then less frequently by locations including the pancreas, hepatobiliary system, ovaries, and testes [10]. The gallbladder is a rare site for NETs, accounting for only $0.04-0.5 \%$ of all NETs $[5,11]$.

In gastroenteropancreatic NETs, it is known that the risk of metastasis largely depends on tumor size [12-14]. As for the gallbladder, Albeores-Saavedra et al. [15] reported that carcinoid tumors of the gallbladder measuring less than $0.5 \mathrm{~cm}$ had no clinical significance. Porter et al. [16] reported that only $6 \%$ of carcinoid tumors of the gallbladder smaller than $1 \mathrm{~cm}$ metastasized, whereas $70 \%$ of tumors $2 \mathrm{~cm}$ or larger were associated with metastasis. Yokoyama et al. [17] reported that two of seven cases $(28.6 \%)$ of carcinoid tumors less than $1 \mathrm{~cm}$ had metastasis at presentation, whereas all five cases measuring $3 \mathrm{~cm}$ or more had metastasis. Although the histologic definition of carcinoid tumor differs from that of NETs, these data indicate that the metastatic risk of NETs of the gallbladder is also size-dependent.

The WHO 2010 classification divides NETs into three categories according to mitotic count and Ki67 labeling index: well-differentiated, low-grade (NET G1); well-differentiated, intermediate-grade (NET G2); and poorly differentiated, high grade (NET G3 or NEC). This classification enables prognostically relevant patient stratification, and NET G1 is thought to be a relatively "benign" category. However, in our case, a tiny gallbladder NET G1 was associated with nodal involvement. To the best of our knowledge, this is the first report that describes a case of gallbladder NET G1, smaller than $1 \mathrm{~cm}$, with nodal involvement. Our experience suggests that, even when smaller than $1 \mathrm{~cm}$ in diameter, NET G1 of the gallbladder can metastasize. 


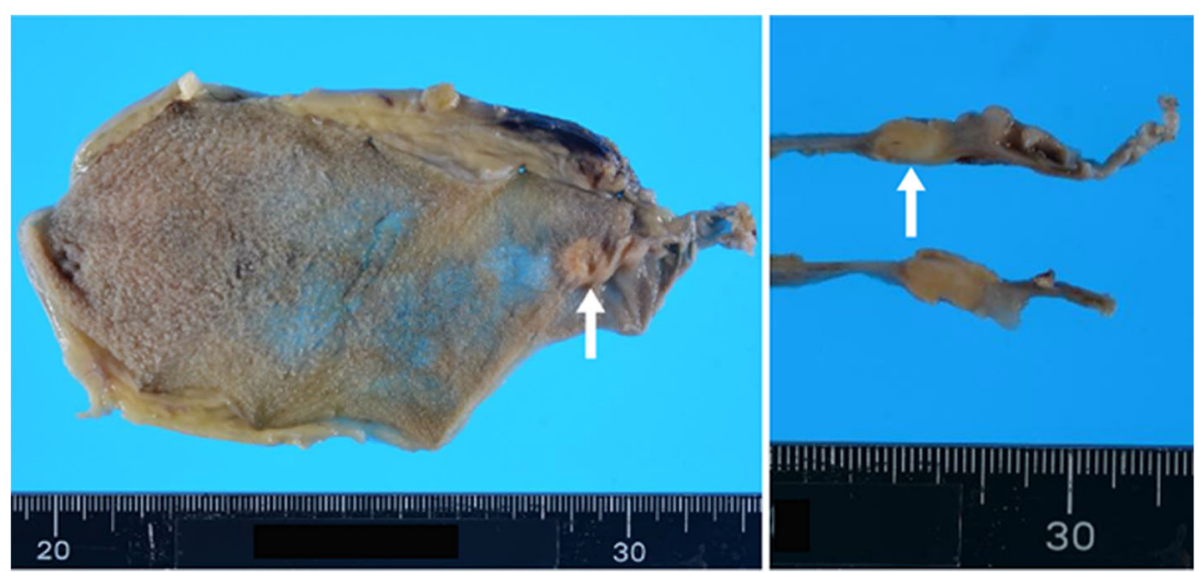

Fig. 2 Surgically resected specimen: a yellowish submucosal tumor $(0.8 \times 0.8 \mathrm{~cm})$ located in the neck of the gallbladder

Preoperative diagnosis of NETs of the gallbladder is rare because, in general, the patients present with non-specific symptoms such as upper abdominal pain, discomfort, jaundice, and weight loss [18]. Previous studies showed that few cases of gallbladder NETs manifested with carcinoid syndrome $[16,19]$. Due to the lack of specific symptoms, the majority of NETs of the gallbladder are identified incidentally after cholecystectomy for cholecystolithiasis [19]. When they are identified in the gallbladder upon removal for presumed benign disease, detailed pathologic examination of the cystic duct node, when found, should be performed to guide whether a radical second resection with regional lymphadenectomy is appropriate. In cases with metastasis in cystic duct nodes, like ours, radical second resection with lymph node dissection should be considered as an option for accurate nodal evaluation, based on the reported outcomes of incidental gallbladder cancer [20].

NET cells have eosinophilic to amphophilic cytoplasm. Rare cases, however, have rich clear cytoplasm and are described as clear cell variants. The clear cell NETs of the pancreas have been reported to be a manifestation of

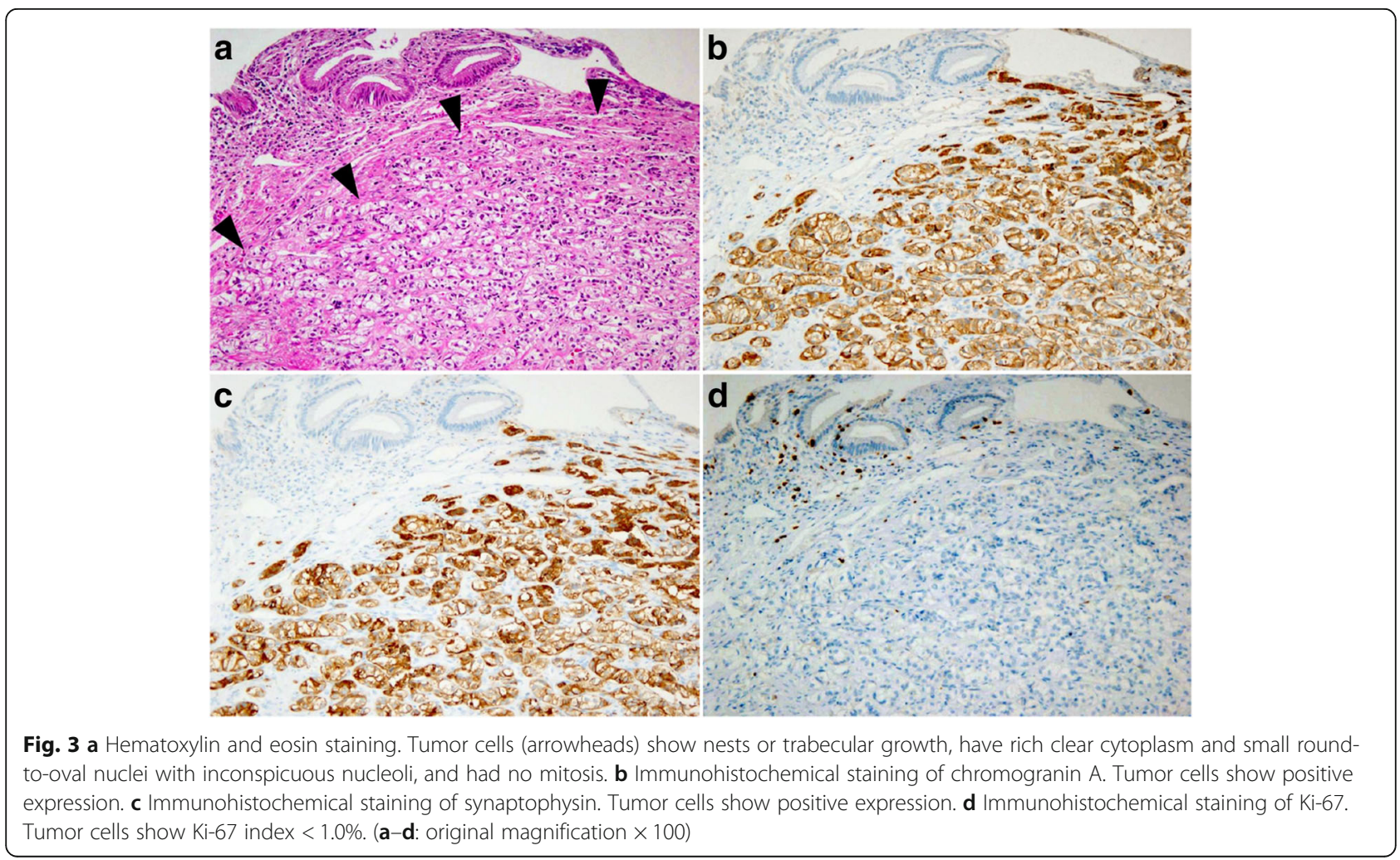


VHL disease [21]. Only three cases of clear cell variant of gallbladder NETs have been described in the literature, one associated with VHL disease [4] and the others not associated with it $[22,23]$. Our case is the third reported case in the literature that describes a clear cell NET of the gallbladder without VHL disease. It would appear that clear cell NETs of the gallbladder are not closely associated with VHL disease.

\section{Conclusions}

Gallbladder NET G1, even when smaller than $1 \mathrm{~cm}$, can metastasize. When NET G1 is incidentally identified in a gallbladder surgical specimen, detailed pathologic examination of the cystic duct node, when found, should be performed to guide whether a radical second resection with regional lymphadenectomy is appropriate.

\section{Abbreviations}

CT: Computed tomography; NET: Neuroendocrine tumors; VHL: Von HippelLindau; WHO: World Health Organization

\section{Acknowledgements}

We are grateful to Eiichi Konishi, MD, PhD, Department of Surgical Pathology, Kyoto Prefectural University of Medicine for assistance with the pathological diagnosis. This case was reported in part as a poster at Joint Congress of the 6th Biennial Congress of the Asian-Pacific Hepato-Pancreato-Biliary Association \& the 29th Meeting of Japanese Society of Hepato-Biliary-Pancreatic Surgery.

\section{Availability of data and materials}

The datasets obtained and/or analyzed during the current study are available from the corresponding author on reasonable request.

\section{Authors' contributions}

All authors were involved in the preparation of this manuscript. YH analyzed the data and wrote the manuscript. YH, JS, and KE designed the study. MT and TS performed the pathological diagnosis. RS, HI, TK, and KY collected the patient's clinical data. JS, MT, and TW revised the manuscript. All authors read and approved the final manuscript.

\section{Ethics approval and consent to participate}

Not applicable.

\section{Consent for publication}

Not applicable.

\section{Competing interests}

The authors declare that they have no competing interests.

\section{Publisher's Note}

Springer Nature remains neutral with regard to jurisdictional claims in published maps and institutional affiliations.

\footnotetext{
Author details

'Division of Digestive and General Surgery, Niigata University Graduate School of Medical and Dental Sciences, Niigata 951-8510, Japan. 2Department of Digestive Surgery, Akita Kousei Medical Center, Akita, Japan. ${ }^{3}$ Department of Diagnostic Pathology, Akita Kousei Medical Center, Akita, Japan
}

Received: 4 March 2018 Accepted: 19 July 2018 Published online: 23 July 2018

\section{References}

1. Yao JC, Hassan M, Phan A, Dagohoy C, Leary C, Mares JE, et al. One hundred years after "carcinoid": epidemiology of and prognostic factors for neuroendocrine tumors in 35,825 cases in the United States. J Clin Oncol. 2008:26:3063-72.

2. Chen $H$, Shen $Y Y, N i X Z$. Two cases of neuroendocrine carcinoma of the gallbladder. World J Gastroenterol. 2014;20:11916-20.

3. Godwin JD 2nd. Carcinoid tumors. An analysis of 2,837 cases. Cancer. 1975; 36:560-9.

4. Sinkre PA, Murakata L, Rabin L, Hoang MP, Albores-Saavedra J. Clear cell carcinoid tumor of the gallbladder: another distinctive manifestation of von Hippel-Lindau disease. Am J Surg Pathol. 2001;25:1334-9.

5. Hough DM, Stephens DH, Johnson CD, Binkovitz LA. Pancreatic lesions in von Hippel-Lindau disease: prevalence, clinical significance, and CT findings. AJR Am J Roentgenol. 1994;162:1091-4.

6. Komminoth P, Arnold R, Capella C, Klimstra DS, Kloppel G. Neuroendocrine neoplasms of the gallbladder and extrahepatic bile ducts. In: Bosman FT, Carneiro F, Hruban $\mathrm{RH}$, Theise HD, editors. WHO classification of tumours of the digestive system. Lyon: International Agency for Research on Cancer; 2010. p. 274-6.

7. Rindi G, Wiedenmann B. Neuroendocrine neoplasms of the gut and pancreas: new insights. Nat Rev Endocrinol. 2011:8:54-64.

8. Soga J. Primary endocrinomas (carcinoids and variant neoplasms) of the gallbladder. A statistical evaluation of 138 reported cases. J Exp Clin Cancer Res. 2003:22:5-15.

9. Oberndorfer S. Karzinoide Tumoren des Dunndarms. Frankf Z Pathol. 1907;1: 426-32. (in German)

10. Gustafsson BI, Kidd M, Modlin IM. Neuroendocrine tumors of the diffuse neuroendocrine system. Curr Opin Oncol. 2008:20:1-12.

11. Nishigami T, Yamada M, Nakasho K, Yamamura M, Satomi M, Uematsu K, et al. Carcinoid tumor of the gall bladder. Intern Med. 1996;35:953-6.

12. Saund MS, Al Natour RH, Sharma AM, Huang Q, Boosalis VA, Gold JS. Tumor size and depth predict rate of lymph node metastasis and utilization of lymph node sampling in surgically managed gastric carcinoids. Ann Surg Oncol. 2011;18:2826-32.

13. Al Natour RH, Saund MS, Sanchez VM, Whang EE, Sharma AM, Huang Q, et al. Tumor size and depth predict rate of lymph node metastasis in colon carcinoids and can be used to select patients for endoscopic resection. J Gastrointest Surg. 2012;16:595-602.

14. Regenet N, Carrere N, Boulanger G, de Calan L, Humeau M, Arnault V, et al. Is the 2 -cm size cutoff relevant for small nonfunctioning pancreatic neuroendocrine tumors: a French multicenter study. Surgery. 2016;159:901-7.

15. Albores-Saavedra J, Henson DE, Klimstra DS, editors. Tumors of the gallbladder, extrahepatic bile ducts, and vaterian system. Atlas of tumor pathology. Washington, DC: Armed Forces Institute of Pathology; 2015. p. 171-84.

16. Porter JM, Kalloo AN, Abernathy EC, Yeo CJ. Carcinoid tumor of the gallbladder: laparoscopic resection and review of the literature. Surgery. 1992;112:100-5

17. Yokoyama Y, Fujioka S, Kato K, Tomono H, Yoshida K, Nimura Y. Primary carcinoid tumor of the gallbladder: resection of a case metastasizing to the liver and analysis of outcomes. Hepato-Gastroenterology. 2000:47:135-9.

18. Buscemi S, Orlando E, Damiano G, Portelli F, Palumbo VD, Valentino A, et al. "Pure" large cell neuroendocrine carcinoma of the gallbladder. Report of a case and review of the literature. Int J Surg. 2016;28(suppl 1):S128-32.

19. Eltawil KM, Gustafsson Bl, Kidd M, Modlin IM. Neuroendocrine tumors of the gallbladder: an evaluation and reassessment of management strategy. J Clin Gastroenterol. 2010;44:687-95.

20. Wakai T, Shirai Y, Hatakeyama K. Radical second resection provides survival benefit for patients with $\mathrm{T} 2$ gallbladder carcinoma first discovered after laparoscopic cholecystectomy. World J Surg. 2002;26:867-71.

21. Hoang MP, Hruban RH, Albores-Saavedra J. Clear cell endocrine pancreatic tumor mimicking renal cell carcinoma: a distinctive neoplasm of von Hippel-Lindau disease. Am J Surg Pathol. 2001;25:602-9.

22. Konishi E, Nakashima Y, Smyrk TC, Masuda S. Clear cell carcinoid tumor of the gallbladder. A case without von Hippel-Lindau disease. Arch Pathol Lab Med. 2003;127:745-7.

23. Ishida M, Shiomi H, Naka S, Tani T, Okabe H. Clear cell neuroendocrine tumor G1 of the gallbladder without von Hippel-Lindau disease. Oncol Lett. 2012:4:1174-6. 